\title{
Duplicate Detection Models for Bug Reports of Software Triage Systems: A Survey
}

\author{
Behzad Soleimani Neysiani* and Seyed Morteza Babamir \\ Department of Software Engineering, University of Kashan, Iran
}

*Corresponding author: Department of Software Engineering, Faculty of Computer \& Electrical Engineering, University of Kashan, Kashan, Esfahan, Iran

\begin{abstract}
Duplicate bug report detection (DBRD) is one of the significant problems of software triage systems, which receive end-user bug reports. DBRD needs automation using artificial intelligence techniques like information retrieval, natural language processing, text and data mining, and machine learning. There are two models of duplicate detection as follows: The first model uses machine learning techniques to learn the features of duplication between pairs of bug reports.

The second model called IR-based that use a similarity metric like REP or BM25F to rank top-k bug reports that are similar to a target bug report. The IR-based approach has identical behavior like the k-nearest neighborhood algorithm of machine learning. This study reviews a decade of duplicate detection techniques and their pros and cons. Besides, the metrics of their validationperformance will be studied.
\end{abstract}

Keywords: Duplicate Detection Model; Machine Learning; Bug Report

\section{Introduction}

Nowadays, software triage systems (STS) like Bugzilla are an impartible tool for huge projects -especially open source- like Open Office, Mozilla Firefox, Eclipse, Android, and so on. The main task of STS is to help the development team for the maintenance phase and get end-user requests like bug reports and suggestions and deal with them. There exist many important tasks for software triage systems like prioritizing bug reports, detecting duplicates, assigning bug reports to developers, track the status of bug reports until they can be fixed [1]. Every bug report consists of various data fields (DF) which can be categorized as follows:

I. Identical DFs like unique identity of bug report, identity of its master bug report which this bug report is duplicate and similar to that one, identity of developer which is responsible to deal with this bug report.

II. Categorical DFs such as company, product, component, and status of bug report which are grouping the bug report in specific categories.
III. Textual DFs contain the main end-user request which is described as a text message in short or long description, e.g., title or description.

IV. Temporal DFs show the Date Time of reporting, assigning, solving and other events about the bug report. Since there are about $30 \%-60 \%$ duplicate bug reports in a STS [2,3], automatic duplicate bug report detection (ADBRD) is one of major problems of STSs. ADBRD needs artificial intelligence techniques like information retrieval, natural language processing, machine learning, text, and data mining. This study focuses on methods of ADBRD and review its methodologies, compare them, and suggest their potential usage [4].

\section{Methodologies of Automatic Duplicate Bug Report Detection}

There are two major methodologies for automatic duplicate bug report detection (ADBRD): 


\section{Information retrieval (IR)-based methodology of automatic duplicate bug report detection (ADBRD)}

The first methodology called the information retrieval-based approach, which its procedure is shown in Figure 1. In the first box, the raw dataset of bug reports exists which should be preprocessed in box 2 till deal with null values, unify the data type of some fields like version and priority and preferably change them to numerical, remove stop words from textual fields, stemming textual fields, correcting the typos in textual DFs $[5,8]$, and make them ready for comparison as box 3 . Then in box 4 , every bug report can be selected as a target bug report, which its duplicates should be found. Usually, the target bug report is a new bug report which is created newly. Then target bug report of box 5 should be compared with other bug reports. Almost all data fields of bug reports cannot be simply compared with an equal operator, especially textual data fields, so, some feature extraction methods are required to calculate their similarity. There are many feature extraction methods based on various data fields like equality operator for nominal categorical DFs, difference or subtract operator for temporal and numerical categorical DFs, information retrieval-based operators like term frequency and inversed document frequency of each term for textual DFs, contextual features which show the similarity of bug report to a special context [9], and so on [1,4]. The feature extraction phase of box 6 returns a numerical vector consist of many similarity metrics as box 7 . Now, the similarity of vectors should be calculated using cosine, Manhattan, Jaccard, BLEU, dice, and same formulas to find top similar bug reports as box 8 . There are many heuristic similarity metrics and semi-heuristic learning algorithms in this phase, like REP [10], MSWGW [11], and Topic Sim [12]. We know the real duplication status of bug reports based on the Table 1: Modes of the duplicate Detection. merge identity data field of bug reports, and now, we can check our prediction, which was true or not in box 9. Then the evaluation of the methodology can be done, and validation performance metrics can be measured in box 10, which its results show the validity performance metrics in box 13. Four modes can be held based on the real status of a bug report, and our prediction, which are shown in Table 1. The validation performance metrics are calculated based on these four modes. The true-positive rate (TPR), true-negative rate (TNR), false-positive rate (FPR), and false-negative rate (FNR) are calculated based on the four modes of Table 1 as (1). In addition, another famous validation metric is accuracy as (2) that show ration of true prediction based on total pairs of bug reports. The Precision metric as (3) is the ratio of true duplicate predicted on the total duplicate predicted. The recall ratio (4) is the fraction of true duplicate predicted based on total actual duplicates. The F1measure as (5) is a harmonic average of Precision and recall.

$$
\begin{aligned}
& T P R=\frac{T D}{T T} T N R=\frac{T N D}{T T} F P R=\frac{F D}{T T} \quad F N R=\frac{F N D}{T T} \\
& \text { Accuracy }=\frac{\text { True Prediction }(T P)}{\operatorname{Total}(T T)}
\end{aligned}
$$$$
\text { Precision }=\frac{\text { TrueDuplicates }(T D)}{\text { PredictedDuplicates }}
$$

$$
\begin{aligned}
& \text { Recall }=\frac{T D}{\text { ActualDuplicates }=T D+F N D} \\
& F 1-\text { measure }=\frac{2 \times \text { Precision } \times \text { Recall }}{\text { Precision }+ \text { Recall }}
\end{aligned}
$$

\begin{tabular}{|c|c|c|c|}
\hline Predict $\downarrow /$ Actual $\rightarrow$ & Actual Dup (AD) $=$ TD+FND & Actual Non-Dup (AND) = FD+TND & Total \\
\hline Predicted Dup & True Dup (TD) & False Dup (FD) & True Prediction (TP=TD+TND) \\
\hline Predicted Non-Dup & False Non-Dup (FND) & True Non-Dup (TND) & False Prediction (FP=FD+FND) \\
\hline Total & & & Total (TT $=$ TP+FP=AD+AND) \\
\hline
\end{tabular}

Machine learning (ML)-based methodology of automatic duplicate bug report detection (ADBRD)

The process of ML-based ADBRD is shown in Figure 2. The boxes 1 to 3 are similar to (Figure 1), but in this case, after building the ready dataset, some pairs of bug reports are selected in box 4 , and the selected pairs in box 5 are used to extract various features in box 6 . Every pair consist of numerical comparison features and a label with two modes: duplicate or non-duplicate in box 7. Now, the features vectors are divided into two separated sets called train and test. The train set is used to learn a machine learner like decision tree, neural network, deep learner, Naïve Bayes, linear regression, and so on in box 8. The built ML is a duplicate finder which is learned the features of duplication. Then the duplicate finder model of box 9 can be used to predict the test set in box 10, and the predicted status of box 11 can be used as (Table 1) to evaluate the validation performance metric as Eqs. (1) to (5). Finally, there are some hybrid approaches that used both IR-based and ML-based methods. The review of related works in state-of-the-art, which used these two types of ADBRD are tabulated in (Table 2). 


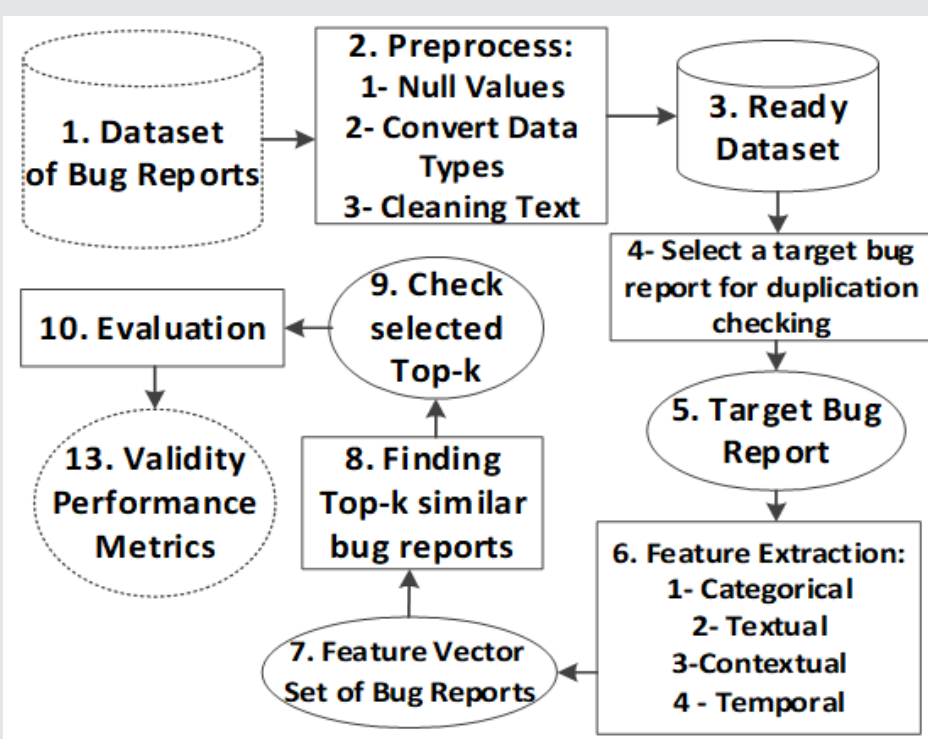

Figure 1: The methodology of duplicate bug report detection using information retrieval techniques.

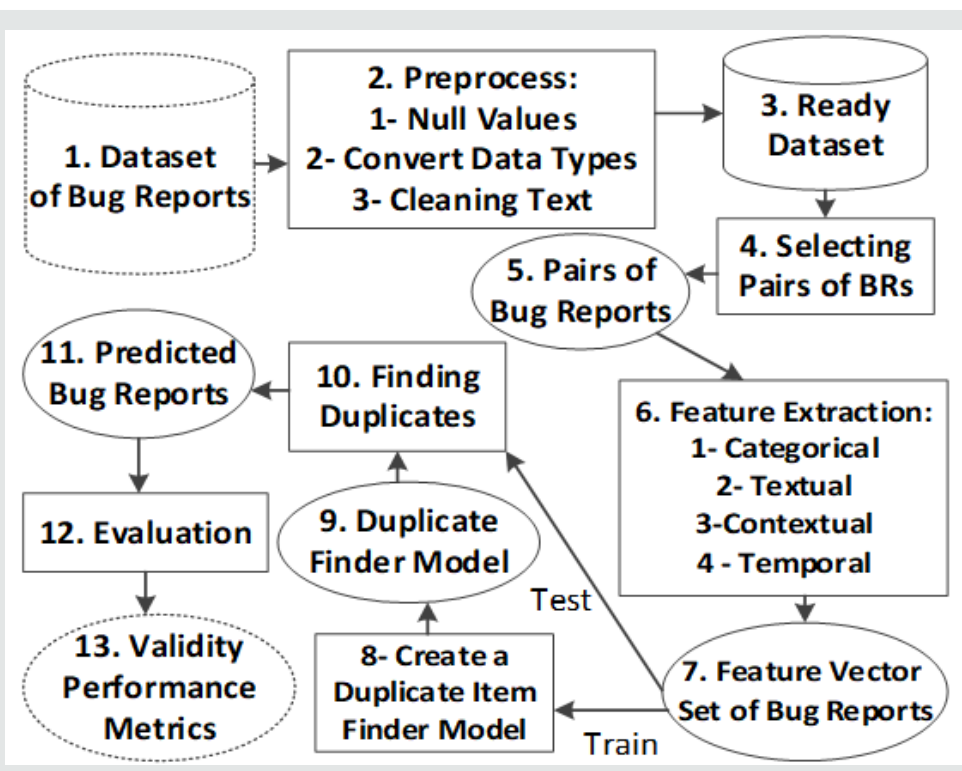

Figure 2: The methodology of duplicate bug report detection using machine learning techniques.

Table 2: Review of duplicate detection models and their metrics.

\begin{tabular}{|c|c|c|c|c|c|c|c|c|c|c|c|}
\hline \multirow[t]{2}{*}{ Row } & \multirow[t]{2}{*}{ Ref } & \multirow[t]{2}{*}{ Year } & \multicolumn{2}{|c|}{$\begin{array}{c}\text { Duplicate Detection } \\
\text { Model }\end{array}$} & \multicolumn{7}{|c|}{ Metrics of Validation Performance } \\
\hline & & & ML-based & IR-based & Recall & Precision & Accuracy & $\begin{array}{l}\text { F1- } \\
\text { measure }\end{array}$ & $\begin{array}{c}\text { TPR, } \\
\text { TNR, } \\
\text { FPR, FNR }\end{array}$ & $\begin{array}{l}\text { Other } \\
\text { Metrics }\end{array}$ & $\begin{array}{c}\text { Incomplete } \\
\text { Validation }\end{array}$ \\
\hline 1. & Hiew [13] & 2006 & & $*$ & $*$ & $*$ & & & $*$ & & \\
\hline 2. & $\begin{array}{c}\text { Runeson et al. } \\
{[14]}\end{array}$ & 2007 & & $*$ & $*$ & & & & & & \\
\hline 3. & $\begin{array}{l}\text { Jalbert and } \\
\text { Weimer [15] }\end{array}$ & 2008 & & $*$ & $*$ & & & & & & \\
\hline 4. & $\begin{array}{l}\text { Bettenburg, et } \\
\text { al. [16] }\end{array}$ & 2008 & & $*$ & $*$ & $*$ & $*$ & & & & \\
\hline 5. & $\begin{array}{l}\text { Bettenburg, et } \\
\text { al. [17] }\end{array}$ & 2008 & $*$ & & & & * & & & & \\
\hline 6. & Wang, et al. [18] & 2008 & & $*$ & $*$ & & & & & & \\
\hline
\end{tabular}




\begin{tabular}{|c|c|c|c|c|c|c|c|c|c|c|c|}
\hline 7. & $\begin{array}{c}\text { Nagwani and } \\
\text { Singh [19] }\end{array}$ & 2009 & & $*$ & & & & & & & * \\
\hline 8. & $\begin{array}{l}\text { Sureka and } \\
\text { Jalote [20] }\end{array}$ & 2010 & & $*$ & * & & & & & & \\
\hline 9. & Sun, et al. [21] & 2010 & $*$ & & $*$ & & & & & & \\
\hline 10. & Sun, et al. [10] & 2011 & & $*$ & * & $*$ & & & & & \\
\hline 11. & Kim, et al. [22] & 2011 & & $*$ & $*$ & $*$ & & $*$ & & & \\
\hline 12. & $\begin{array}{c}\text { Nguyen, et al. } \\
\text { [12] }\end{array}$ & 2012 & $*$ & $*$ & & & $*$ & & & & \\
\hline 13. & $\begin{array}{c}\text { Banerjee, et al. } \\
{[11]}\end{array}$ & 2012 & & $*$ & * & & & & & & \\
\hline 14. & Tian, et al. [23] & 2012 & $*$ & $*$ & & & & $*$ & $*$ & & \\
\hline 15. & Liu, et al. [24] & 2013 & $*$ & & & * & & & $*$ & & \\
\hline 16. & $\begin{array}{c}\text { Alipour, et al. } \\
{[25,26]}\end{array}$ & 2013 & $*$ & & & & $*$ & & $*$ & $\begin{array}{l}\text { Kappa, } \\
\text { AUC }\end{array}$ & \\
\hline 17. & Feng, et al. [27] & 2013 & $*$ & $*$ & $*$ & $*$ & $*$ & & $*$ & & \\
\hline 18. & Lazar, et al. [28] & 2014 & $*$ & & $*$ & $*$ & $*$ & & & AUC & \\
\hline 19. & $\begin{array}{c}\text { Wang, et al. } \\
{[29,30]}\end{array}$ & 2014 & & $*$ & * & * & & & & & \\
\hline 20. & Thung, et al. [31] & 2014 & & $*$ & & & & & & & * \\
\hline 21. & $\begin{array}{l}\text { Gopalan and } \\
\text { Krishna [32] }\end{array}$ & 2014 & & $*$ & & & & $*$ & $*$ & & \\
\hline 22. & $\begin{array}{c}\text { Tsuruda, et al. } \\
\text { [33] }\end{array}$ & 2015 & $*$ & & $*$ & * & $*$ & & & & \\
\hline 23. & $\begin{array}{c}\text { Aggarwal, et al. } \\
{[34,35]}\end{array}$ & $\begin{array}{l}2015, \\
2017\end{array}$ & * & & & & $*$ & & & Карра & \\
\hline 24. & $\begin{array}{l}\text { Sharma and } \\
\text { Sharma [36] }\end{array}$ & 2015 & $*$ & & & & & & $*$ & ROC & \\
\hline 25. & $\begin{array}{l}\text { Hindle, et al. } \\
\text { [37] }\end{array}$ & 2016 & $*$ & & & $*$ & $*$ & & $*$ & $\begin{array}{l}\text { Kappa, } \\
\text { AUC }\end{array}$ & \\
\hline 26. & Hindle $[38,39]$ & 2016 & & $*$ & $*$ & $*$ & & & & MRR & \\
\hline 27. & Zou, et al. [40] & 2016 & & * & $*$ & $*$ & * & & & & \\
\hline 28. & Yang, et al. [41] & 2016 & & $*$ & $*$ & $*$ & & & & MRR & \\
\hline 29. & $\begin{array}{c}\text { Swapna and } \\
\text { Reddy [42] }\end{array}$ & 2016 & & $*$ & & & & & & & * \\
\hline 30. & Lin, et al. [43] & 2016 & $*$ & & $*$ & & & & & & \\
\hline 31. & Pasala, et al. [44] & 2016 & * & $*$ & $*$ & & & & & & \\
\hline 32. & Rakha, et al. [45] & 2016 & $*$ & & $*$ & $*$ & & $*$ & & AUC & \\
\hline 33. & $\begin{array}{c}\text { Panichella, et al. } \\
{[46]}\end{array}$ & 2016 & & $*$ & $*$ & & & & & & \\
\hline 34. & Kang [47] & 2017 & & $*$ & * & & & & & & \\
\hline 35. & $\begin{array}{c}\text { Koochekian } \\
\text { Sabor, et al. [48] }\end{array}$ & 2017 & & * & $*$ & & & & & & \\
\hline 36. & $\begin{array}{l}\text { Deshmukh, et al. } \\
{[49]}\end{array}$ & 2017 & $*$ & & $*$ & * & $*$ & & & & \\
\hline 37. & $\begin{array}{c}\text { Banerjee, et al. } \\
{[50]}\end{array}$ & 2017 & & $*$ & * & & & & $*$ & & \\
\hline 38. & Bagal, et al. [51] & 2017 & $*$ & $*$ & & & & & & & * \\
\hline 39. & Rakha, et al. [52] & 2018 & & $*$ & $*$ & $*$ & & & & & \\
\hline 40. & $\begin{array}{c}\text { Budhiraja, et al. } \\
\text { [53] }\end{array}$ & 2018 & $*$ & & * & & & & & & \\
\hline 41. & $\begin{array}{c}\text { Budhiraja, et al. } \\
{[54]}\end{array}$ & 2018 & & $*$ & * & & & & & & \\
\hline 42. & Su and Joshi [55] & 2018 & $*$ & & * & & & & & & \\
\hline
\end{tabular}




\begin{tabular}{|c|c|c|c|c|c|c|c|c|c|c|}
\hline 43. & $\begin{array}{l}\text { Bommaraju, et } \\
\text { al. [56] }\end{array}$ & 2018 & & $*$ & & & & & & * \\
\hline 44. & $\begin{array}{c}\text { Santos, et al. } \\
\text { [57] }\end{array}$ & 2018 & & $*$ & $*$ & $*$ & & $*$ & & \\
\hline 45. & Chen, et al. [58] & 2018 & & $*$ & $*$ & $*$ & & & MRR & \\
\hline 46. & Phuc [59] & 2018 & & $*$ & $*$ & & & & & \\
\hline 47. & Xie, et al. [60] & 2018 & $*$ & & & & $*$ & $*$ & & \\
\hline 48. & $\begin{array}{c}\text { Soleimani } \\
\text { Neysiani and } \\
\text { Babamir [61] }\end{array}$ & 2019 & * & & $*$ & $*$ & * & & & \\
\hline 49. & $\begin{array}{c}\text { Soleimani } \\
\text { Neysiani and } \\
\text { Babamir [9] }\end{array}$ & 2019 & $*$ & & $*$ & $*$ & $*$ & & & \\
\hline 50. & $\begin{array}{c}\text { Ebrahimi, et al. } \\
{[62]}\end{array}$ & 2019 & & $*$ & $*$ & $*$ & & & & \\
\hline 51. & LEE, et al. [63] & 2019 & & $*$ & & & & & & * \\
\hline 52. & $\begin{array}{c}\text { Poddar, et al. } \\
\text { [64] }\end{array}$ & 2019 & & $*$ & * & $*$ & & $*$ & & \\
\hline 53. & $\begin{array}{c}\text { Chaparro, et al. } \\
\text { [65] }\end{array}$ & 2019 & & $*$ & $*$ & & & & & \\
\hline 54. & $\begin{array}{c}\text { Soleimani } \\
\text { Neysiani and } \\
\text { Babamir [66] }\end{array}$ & 2019 & $*$ & - & $*$ & $*$ & $*$ & - & - & - \\
\hline
\end{tabular}

\section{Conclusion}

This study reviews the methodologies of automatic duplicate bug report detection (ADBRD), including information retrieval (IR)-based approach and machine learning (ML)-based approach. The IR-based approach is mostly used for online ADBRD and MLbased in used for offline application, even though both of them can be used for online and offline applications. Also, IR-based approach behavior is similar to k-nearest neighbor (k-NN) algorithm of machine learning which makes this approach be a special case in ML-based approach, but, the most analysis of IR-based approach on the details of parameter $\mathrm{K}$ in k-NN make this approach famous and isolate, especially it seems many authors were not familiar with k-NN algorithm, so, they insist on implementing its detail their selves with custom modification in selecting bug reports for comparison or changing the similarity metric and introduce new heuristic similarity formulas. Some studies use a combination of both approaches. Because the parameters of experiments in stateof-the-art are different, it is difficult to judge which approach is more useful and accurate. Finally, future work is to find an accurate and fast ADBRD.

\section{References}

1. Soleimani Neysiani B, Babamir SM (2016) Methods of Feature Extraction for Detecting the Duplicate Bug Reports in Software Triage Systems. presented at the International Conference on Information Technology, Communications and Telecommunications (IRICT), Tehran, Iran.

2. Cavalcanti YC, Neto PAMS, Lucrédio D, Vale T, de Almeida ES, et al. (2013) The bug report duplication problem: an exploratory study. Software Quality Journal 21(1): 39-66.
3. Zhang J, Wang X, Hao D, Xie B, Zhang L, et al. (2015) A survey on bugreport analysis. Science China Information Sciences 58(2): 1-24.

4. Aminoroaya Z, Soleimani Neysiani B, Nadimi Shahraki MH (2018) Detecting Duplicate Bug Reports Techniques. Research Journal of Applied Sciences 13(9): 522-531.

5. Soleimani Neysiani B, Babamir SM (2018) Automatic Typos Detection in Bug Reports. presented at the IEEE 12th International Conference Application of Information and Communication Technologies, Kazakhstan.

6. Soleimani Neysiani B, Babamir SM (2019) Automatic Interconnected Lexical Typo Correction in Bug Reports of Software Triage Systems. presented at the International Conference on Contemporary Issues in Data Science, Zanjan, Iran.

7. Soleimani Neysiani B, Babamir SM (2019) Fast Language-Independent Correction of Interconnected Typos to Finding Longest Terms Using Trie for Typo Detection and Correction. presented at the 24th International Conference on Information Technology (IVUS), Lithuania.

8. Soleimani Neysiani B, Babamir SM (2019) New labeled dataset of interconnected lexical typos for automatic correction in the bug reports. SN Applied Sciences 1(11): 1385.

9. Soleimani Neysiani B, Babamir SM (2019) New Methodology of Contextual Features Usage in Duplicate Bug Reports Detection. IEEE 5th International Conference on Web Research (ICWR), Tehran, Iran.

10. Sun C, Lo D, Khoo SC, Jiang J (2011) Towards more accurate retrieval of duplicate bug reports. In Proceedings of the 26th IEEE/ACM International Conference on Automated Software Engineering (ASE) pp. 253-262.

11. Banerjee S, Cukic B, Adjeroh D (2012) Automated duplicate bug report classification using subsequence matching. In IEEE 14th International Symposium on High-Assurance Systems Engineering (HASE) pp. 74-81.

12. Nguyen AT, Nguyen TT, Nguyen TN, Lo D, Sun C (2012) Duplicate bug report detection with a combination of information retrieval and topic modeling. In Proceedings of the 27th IEEE/ACM International Conference on Automated Software Engineering (ASE) pp. 70-79. 
13. Hiew L (2006) Assisted detection of duplicate bug reports. Master of Science, Faculty of Graduate Studies(Computer Science), The University of British Columbia, Canada.

14. Runeson P, Alexandersson M, Nyholm O (2007) Detection of duplicate defect reports using natural language processing. In 29th International Conference on Software Engineering (ICSE) pp. 499-510.

15. Jalbert N, Weimer W (2008) Automated duplicate detection for bug tracking systems. In IEEE International Conference on Dependable Systems and Networks (DSN) With FTCS and DCC pp. 52-61.

16. Bettenburg N, Premraj R, Zimmermann T, Kim S (2008) Extracting structural information from bug reports. in Proceedings of the 2008 international working conference on Mining software repositories, Leipzig, Germany pp. 27-30.

17. Bettenburg N, Premraj R, Zimmermann T, Kim S (2008) Duplicate bug reports considered harmful... really ?. in IEEE International Conference on Software Maintenance (ICSM) pp. 337-345.

18. Wang X, Zhang L, Xie T, Anvik J, Sun J (2008) An approach to detecting duplicate bug reports using natural language and execution information. In Proceedings of the 30th international conference on Software engineering, Leipzig, Germany pp. 461-470.

19. Nagwani NK, Singh P (2009) Weight similarity measurement model based, object-oriented approach for bug databases mining to detect similar and duplicate bugs. In Proceedings of the International Conference on Advances in Computing, Communication and Control pp. 202-207.

20. Sureka A, Jalote P (2010) Detecting duplicate bug report using character n-gram-based features. In 17th Asia Pacific Software Engineering Conference (APSEC) pp. 366-374.

21. Sun C, Lo D, Wang X, Jiang J, Khoo SC (2010) A discriminative model approach for accurate duplicate bug report retrieval.In Proceedings of the 32nd ACM/IEEE International Conference on Software Engineering 1: $45-54$.

22. Kim S, Zimmermann T, Nagappan N (2001)Crash graphs: An aggregated view of multiple crashes to improve crash triage. In Dependable Systems \& Networks (DSN), 2011 IEEE/IFIP 41st International Conference pp. 486-493.

23. Tian Y, Sun C, Lo D (2012) Improved duplicate bug report identification In Software Maintenance and Reengineering (CSMR), 2012 16th European Conference pp. 385-390.

24. Liu K, Tan HBK, Chandramohan M (2012) Has this bug been reported? In Proceedings of the ACM SIGSOFT $20^{\text {th }}$ International Symposium on the Foundations of Software Engineering pp. 28.

25. Alipour A, Hindle A, Stroulia E (2013) A Contextual Approach Towards More Accurate Duplicate Bug Report Detection. In Proceedings of the 10th Working Conference on Mining Software Repositories, San Francisco, CA, USA pp. 183-192.

26. Alipour A (2013) A Contextual Approach Towards More Accurate Duplicate Bug Report Detection. Master of Science, Department of Computing Science, University of Alberta, Faculty of Graduate Studies and Research.

27. Feng L, Song L, Sha C, Gong X (2013) Practical duplicate bug reports detection in a large web-based development community. In Asia Pacific Web Conference pp. 709-720.

28. Lazar A, Ritchey S, Sharif B (2014) Improving the accuracy of duplicate bug report detection using textual similarity measures. In MSR 2014 Proceedings of the 11th Working Conference on Mining Software Repositories, Hyderabad, India pp. 308-311.

29. Wang S, Khomh F, Zou Y (2013) Improving bug localization using correlations in crash reports. In 10th IEEE Working Conference on Mining Software Repositories (MSR) pp. 247-256.
30. Wang S, Khomh F, Zou Y (2014) Improving bug management using correlations in crash reports. Empirical Software Engineering 22(2): $1-31$.

31. Thung F, Kochhar PS, Lo D (2014) Dup Finder: integrated tool support for duplicate bug report detection. In Proceedings of the 29th ACM/IEEE international conference on Automated software engineering (ASE), Vasteras, Sweden pp. 871-874.

32. Gopalan RP, Krishna A (2014) Duplicate bug report detection using clustering. in Software Engineering Conference (ASWEC), 2014 23rd Australian pp. 104-109.

33. Tsuruda A, Manabe Y, Aritsugi M (2015) Can We Detect Bug Report Duplication with Unfinished Bug Reports ?. In Asia-Pacific Software Engineering Conference (APSEC) pp. 151-158.

34. Aggarwal K, Rutgers T, Timbers F, Hindle A, Greiner R, et al. (2015) "Detecting duplicate bug reports with software engineering domain knowledge. In IEEE 22nd International Conference on Software Analysis, Evolution and Reengineering (SANER), Montreal pp. 211-220.

35. Aggarwal K, Timbers F, Rutgers T, Hindle A, Stroulia E, et al. (2016) Detecting duplicate bug reports with software engineering domain knowledge. Journal of Software: Evolution and Process 29(3):1821.

36. Sharma A, Sharma S (2015) Bug Report Triaging Using Textual, Categorical and Contextual Features Using Latent Dirichlet Allocation. International Journal for Innovative Research in Science and Technology (IJIRST) 1(9): 85-96.

37. Hindle A, Alipour A, Stroulia E (2016) A contextual approach towards more accurate duplicate bug report detection and ranking. Empirical Software Engineering, journal article 21(2): 368-410.

38. Hindle A (2013) Stopping duplicate bug reports before they start with Continuous Querying for bug reports. PeerJ Preprints pp. 2167-9843.

39. Hindle A, Onuczko C (2018) Preventing duplicate bug reports by continuously querying bug reports. Empirical Software Engineering pp. $1-35$.

40. Zou J, Xu L, Yang M, Zhang X, Zeng J, et al. (2016) Automated Duplicate Bug Report Detection Using Multi-Factor Analysis. IEICE Transactions on Information and Systems 99(7): 1762-1775.

41. Yang X, Lo D, Xia X, Bao L, Sun J (2016) Combining word embedding with information retrieval to recommend similar bug reports. In IEEE 27 th International Symposium on Software Reliability Engineering (ISSRE) pp. 127-137.

42. Swapna D, Reddy KT (2016) Duplicate Bug Report Detection of User Interface Bugs using Decision Tree Induction and Inverted Index Structure. Spreizen International Journal on Science and Technology 2: 26-34.

43. Lin MJ, Yang CZ, Lee CY, Chen CC (2016) Enhancements for duplication detection in bug reports with manifold correlation features. Journal of Systems and Software 121: 223-233.

44. Pasala A, Guha S, Agnihotram G, Prateek BS, Padmanabhuni S (2016) An Analytics-Driven Approach to Identify Duplicate Bug Records in Large Data Repositories. in Data Science and Big Data Computing: Frameworks and Methodologies, Z. Mahmood Ed. Cham: Springer International Publishing pp. 161-187.

45. Rakha MS, Shang W, Hassan AE (2016) Studying the needed effort for identifying duplicate issues. Empirical Software Engineering 21(5): 1960-1989.

46. Panichella A,Dit B, Oliveto R, Penta MD, Poshyvanyk D, et al. (2016) Parameterizing and Assembling IR-Based Solutions for SE Tasks Using Genetic Algorithms. In 2016 IEEE 23rd International Conference on Software Analysis, Evolution, and Reengineering (SANER) 1: 314-325.

47.L Kang (2017) Automated Duplicate Bug Reports Detection-An Experiment at Axis Communication AB. Master of Science, Software 
Engineering, Blekinge Institute of Technology, Faculty of Computing, Sweden.

48. Sabor KK, Hamou Lhadj A, Larsson A (2017) DURFEX: A Feature Extraction Technique for Efficient Detection of Duplicate Bug Reports. In 2017 IEEE International Conference on Software Quality, Reliability and Security (QRS), Prague, Czech Republic IEEE pp. 240-250.

49. Deshmukh J, Podder S, Sengupta S, Dubash N (2017) Towards Accurate Duplicate Bug Retrieval Using Deep Learning Techniques. In 2017 IEEE International Conference on Software Maintenance and Evolution (ICSME), 2017: IEEE pp. 115-124.

50. Banerjee S, Syed Z, Helmick J, Culp M, Ryan K et al. (2017) Automated triaging of very large bug repositories. Information and Software Technology 89: 1-13.

51. Bagal PV, Joshi SA, Chien HD, Diez RR, Woo DC, et al. (2017) Duplicate bug report detection using machine learning algorithms and automated feedback incorporation.

52. Rakha MS, Bezemer CP, Hassan AE (2018) Revisiting the Performance Evaluation of Automated Approaches for the Retrieval of Duplicate Issue Reports. IEEE Transactions on Software Engineering 44(12): 12451268

53. Budhiraja A, Dutta K, Reddy R, Shrivastava M (2018) DWEN: deep word embedding network for duplicate bug report detection in software repositories. In Proceedings of the 40th International Conference on Software Engineering: Companion Proceedings pp. 193-194.

54. Budhiraja A, Reddy R, Shrivastava M (2018) LWE: LDA refined word embeddings for duplicate bug report detection. In Proceedings of the 40th International Conference on Software Engineering: Companion Proceeedings pp. 165-166.

55. Su E, Joshi S (2018) Leveraging product relationships to generate candidate bugs for duplicate bug prediction. In Proceedings of the 40th International Conference on Software Engineering: Companion Proceedings pp. 210-211.

56. Bommaraju SP, Pasala A, Rao S (2018) System and method for detection of duplicate bug reports.

57. Santos I, Araújo J, Lima C, Prudêncio RB, Barros F (2018) AVS: An approach to identifying and mitigating duplicate bug reports. In
Proceedings of the XIV Brazilian Symposium on Information Systems pp. 22.

58. Chen M (2018) Using Document Embedding Techniques for Similar Bug Reports Recommendation. In 2018 IEEE 9th International Conference on Software Engineering and Service Science (ICSESS) pp. 811-814.

59. Phuc NM (2018) Using BM25 weighting and Cluster Shrinkage for Detecting Duplicate Bug Reports. International Journal of Advanced Research in Computer and Communication Engineering 7(11): 71-77.

60. Xie Q Wen Z, Zhu J, Gao C, Zheng Z (2018) Detecting Duplicate Bug Reports with Convolutional Neural Networks. In 2018 25th Asia-Pacific Software Engineering Conference (APSEC) pp. 416-425.

61. Soleimani Neysiani B, Babamir SM (2019) Improving Performance of Automatic Duplicate Bug Reports Detection Using Longest Common Sequence: Introducing New Textual Features for Textual Similarity Detection. In IEEE 5th International Conference on Knowledge-Based Engineering and Innovation (KBEI), Tehran, Iran.

62. Ebrahimi N, Trabelsi A, Islam MS, Hamou Lhadj A, Khanmohammadi K (2019) An HMM-based approach for automatic detection and classification of duplicate bug reports. Information and Software Technology 113: 98-109.

63. Lee J, Kim D, Jung W (2019) Cost-Aware Clustering of Bug Reports by Using a Genetic Algorithm. Journal of Information Science \& Engineering 35(1): 175-200.

64. Poddar L, Neves L, Brendel W, Marujo L, Tulyakov S, et al. (2019) Train One Get One Free: Partially Supervised Neural Network for Bug Report Duplicate Detection and Clustering.

65. Chaparro O, Florez JM, Singh U, Marcus A (2019) Reformulating Queries for Duplicate Bug Report Detection. In 2019 IEEE 26th International Conference on Software Analysis, Evolution and Reengineering (SANER ) pp. 218-229.

66. B. Soleimani Neysiani and S. M. Babamir (2019)"Effect of Typos Correction on the validation performance of Duplicate Bug Reports Detection," presented at the 10th International Conference on Information and Knowledge Technology (IKT), Tehran, Iran, 2020: 1-2.

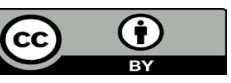

This work is licensed under Creative Commons Attribution 4.0 License

To Submit Your Article Click Here: Submit Article

DOI: $10.32474 /$ CTCSA.2019.01.000123

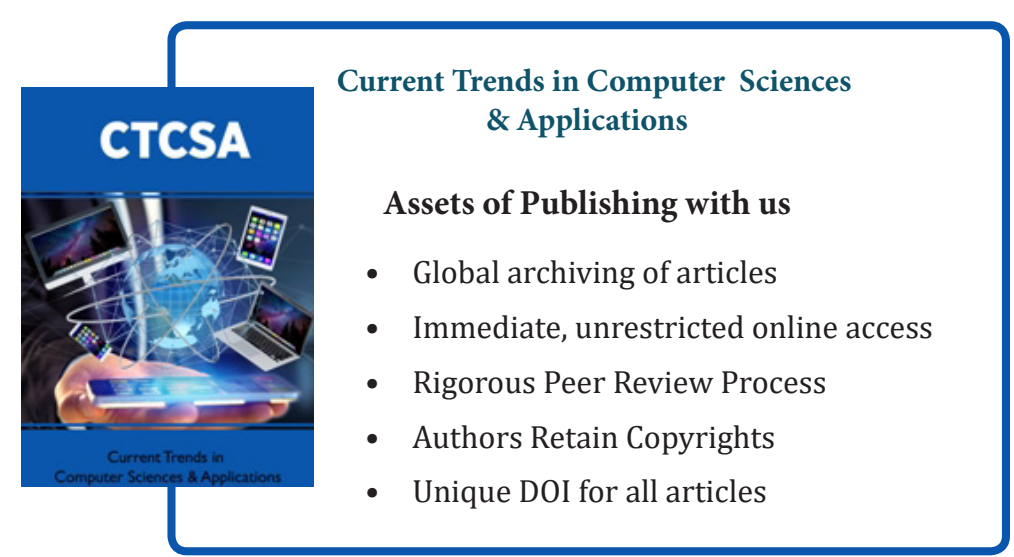

